\title{
In-hospital outcomes of delayed stenting in hemodynamically stable patients with ST-segment elevation myocardial infarction: the CCC (Care for Cardiovascular Disease in China) project
}

\author{
Jia-Wei $\mathrm{Wu}^{1,2}$, Hao $\mathrm{Hu}^{2}$, Dan $\mathrm{Li}^{2}$, Li-Kun $\mathrm{Ma}^{2}$ \\ ${ }^{1}$ School of Medicine, Shandong University, Jinan 370100, China; ${ }^{2}$ Department of Cardiology, The First Affiliated Hospital of USTC, Division of \\ Life Science and Medicine, University of Science and Technology of China, Hefei 230001, China \\ Contributions: (I) Conception and design: JW Wu, LK Ma; (II) Administrative support: LK Ma; (III) Provision of study material or patients: JW \\ Wu, H Hu, D Li, LK Ma; (IV) Collection and assembly of data: JW Wu, H Hu, D Li, LK Ma; (V) Data analysis and interpretation: JW Wu; (VI) \\ Manuscript writing: All authors; (VII) Final approval of manuscript: All authors. \\ Correspondence to: Li-Kun Ma. Department of Cardiology, The First Affiliated Hospital of USTC, Division of Life Science and Medicine, University \\ of Science and Technology of China, Hefei 230001, China. Email: 1kma119@163.com.
}

Background: For hemodynamically stable patients with ST-segment elevation myocardial infarction
(STEMI) who missed the reperfusion window, optimal timing for delayed revascularization remains
controversial.
Methods: We investigated 7,698 consecutive patients without cardiogenic shock, serious heart failure,
or thrombolysis who underwent delayed stenting (12 hours to 28 days after STEMI) at multiple centers
in China. The patients were divided according to delayed PCI timing into very early (12-72 hours), early
(3-7 days), intermediate (7-14 days) and late (14-28 days) groups. The primary outcome was in-hospital rate
of major adverse cardiovascular events (MACE); secondary outcomes were in-hospital rates of all bleeding
events, heart failure and sudden cardiac arrest (SCA). All endpoint events were a composite of the primary
and secondary endpoints.

Results: In-hospital MACE rate was similar among groups $(\mathrm{P}=0.588)$. Patients who underwent late vs. very early, early and intermediate delayed PCI had higher in-hospital rates of secondary events (13\% vs. $8.0 \%, 8.1 \%$ and $0.3 \%, \mathrm{P}<0.001)$ and heart failure (11.8\% vs. $6.2 \%, 6.3 \%$ and $7.6 \%, \mathrm{P}<0.001$, respectively). For all in-hospital events, the late $v s$. intermediate group was at higher risk (OR =1.26, 95\% CI: 1.02 to 1.56, $\mathrm{P}=0.029)$; and in subgroup analysis, patients with Killip class II or III heart failure had similar rates ( $\mathrm{OR}=1.02$, 95\% CI: 0.74 to $1.40, \mathrm{P}=0.908)$; while women $(\mathrm{OR}=1.67,95 \% \mathrm{CI}: 1.07$ to $2.62, \mathrm{P}=0.024)$, and smokers ( $\mathrm{OR}=1.46,95 \% \mathrm{CI}: 1.05$ to $2.02, \mathrm{P}=0.023$ ) had higher rates.

Conclusions: Late delayed PCI (14-28 days) after STEMI was associated with a higher incidence of inhospital adverse events particularly in women and smokers but not with Killip class II-III heart failure, which might allow medical treatment to improve function.

Keywords: Percutaneous intervention; ST-segment elevation myocardial infarction (STEMI); Killip class

Submitted Jun 29, 2019. Accepted for publication Aug 09, 2019.

doi: $10.21037 / c d t .2019 .08 .10$

View this article at: http://dx.doi.org/10.21037/cdt.2019.08.10

\section{Introduction}

Early reperfusion of coronary flow, i.e., within 12 hours from symptom onset by primary percutaneous coronary intervention (PPCI) as per current guidelines, significantly reduces infarct size propitiating infarcted myocardial regeneration and left ventricular (LV) remodeling (1-3). However, wiring, thrombus aspiration and balloon dilation during PPCI favor a thrombotic environment within most 


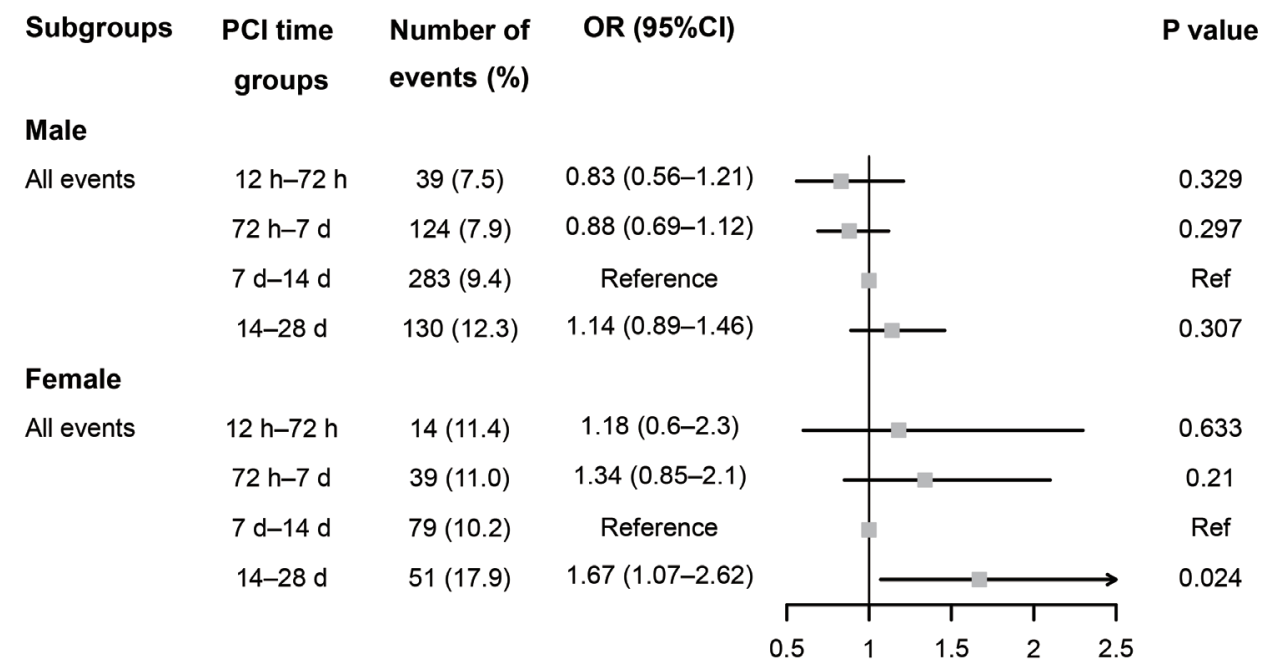

Figure 1 Subgroup analyses for all in-hospital events by sex.

infarct-related arteries (IRA) especially within 12-48 hours of STEMI, which might result in immediate microvascular obstruction (MVO) with unfavorable prognosis $(4,5)$. Several studies demonstrated that patients with an invasive approach ( $>12$ hours) after STEMI had better clinical outcomes than patients with conservative treatment, which supports the idea of late reperfusion of STEMI (6-9). However, in the Occluded Artery Trial (OAT), routine PCI for a totally occluded IRA 3-28 days after acute $\mathrm{MI}$ failed to reduce five-year mortality, reinfarction, and severe heart failure rates (10). Because the best time to perform delayed revascularization remains controversial for patients who missed the golden revascularization time for STEMI, the present study aimed to assess in-hospital outcomes of delayed stenting 12 hours to 28 days after STEMI symptom onset.

\section{Methods}

\section{Study population}

Of the 38,036 patients with STEMI (defined as STsegment elevation consist with infarction of $\geq 2 \mathrm{~mm}$ in contiguous chest leads and/or ST-segment elevation $\geq 1 \mathrm{~mm}$ in $\geq 2$ standard leads or new left bundle branch block, and positive cardiac necrosis markers) enrolled from November 2014 to June 2017 in the CCC (Care for Cardiovascular disease in China) project, the present study retrospectively analyzed data from 7,698 patients who underwent delayed stenting, i.e., at $>12$ hours after symptom onset, and without cardiogenic shock, serious heart failure or thrombolysis,. The CCC project is an ongoing, nationwide, multicenter, cohort study, which is run by the American Heart Association (AHA) in collaboration with the Chinese Society of Cardiology (CSC). One hundred forty-five hospitals have been invited to participate in this project. All the hospitals have their own cardiology unit, a cardiology surgical unit, an internal medicine unit and perform $\geq 50$ primary percutaneous intervention (PCI) per year. Data were collected from electronic case report forms by skilled physicians who were familiar with every patient. The study was approved by the Institutional Review Boards of participating hospitals and all patients provided written informed consent.

Patients between 18 and 85 years old, diagnosed with STEMI and who missed PPCI (within 12 hours) were included, and those with cardiogenic shock or serious heart failure for whom the recommended window for invasive coronary revascularization exceeded 12 hours, or patients treated with primary thrombolysis or delayed stenting (>28 day) were excluded.

All enrolled patients were divided according to delayed PCI timing after STEMI into 4 groups: very early (within 12 to 72 hours), early ( $3-7$ days), intermediate (7-14 days) and late (14-28 days) (Figure 1).

\section{Study outcomes}

The primary in-hospital outcome was rate of major adverse cardiovascular events (MACE), including death from any reason, cardiac death, recurrent myocardial infarction 
(MI), stent thrombosis and ischemic stroke. The secondary outcomes were rates of all bleeding events, heart failure and sudden cardiac arrest during hospitalization. All endpoint events were a composite of the primary and secondary endpoints.

\section{Definition of events}

Cardiac death was defined as death not clearly due to a noncardiac reason. Myocardial reinfarction was defined as recurrent symptoms with a new onset of ST-elevation or a complete left bundle branch block or with at least $20 \%$ revelation of CK-MB between two assays. Stent thrombosis was defined as Definite or confirmed event (symptoms suggestive of an acute coronary syndrome and angiographic or pathologic confirmation of stent thrombosis). Heart failure was defined as the presence of a third heart sound, New York Heart Association class, dyspnea, or evidence of pulmonary congestion on chest radiograph. SCA is a condition in which the heart suddenly and unexpectedly stops beating.

\section{Statistical analysis}

Means (standard deviation) and number (proportions) were calculated for characteristics according to PCI timing categories. To compare characteristics of the study subjects between PCI timing categories, analysis of variance was used for continuous variables and chi-squared or KruskalWallis rank-sum test for categorical variables. We used multiple unconditional logistic regression analyses to estimate the relationship between PCI timing and all, primary, and secondary events with adjustment for potential confounders, and odd ratios (OR) and $95 \%$ confidence intervals (95\% CI) were calculated. Regression models for different clinical subgroups were also conducted. All $\mathrm{P}$ values were 2-tailed, with a significance level of 0.05 . Data management and all analyses were performed using $\mathrm{R}$ software program, version 3.4.3 (http://www.R-project.org).

\section{Results}

A total of 63,641 patients with acute coronary syndrome was hospitalized in 145 hospitals from November 2014 to June 2017. Among the latter population, 38,036 patients presented with STEMI, of whom 7,698 underwent delayed PCI (12 hours-28 days after STEMI onset) and were included in the present study. A comparison of clinical characteristics and in-hospital outcomes among the 4 study groups by delayed PCI timing is shown in Table 1. Length of stay (LOS) differed among groups, with the longest LOS in late group (7.3 days). The intermediate and very early groups had the greatest proportion of smokers (49.9\%) and of patients with cerebrovascular disease history (16.5\%), respectively. Although the highest in-hospital all events rate was observed in the late group (13.5\%), there was no significant difference in the rate of primary events among the 4 groups $(\mathrm{P}=0.588)$. During hospitalization, patients who underwent late PCI had higher rates of secondary events and heart failure than those who received very early, early and intermediate delayed PCI (secondary events: $13.0 \%$ versus $8.0 \%, 8.1 \%$ and $9.3 \%, \mathrm{P}<0.001$; heart failure: $11.8 \%$ versus $6.2 \%, 6.3 \%$ and $7.6 \%, \mathrm{P}<0.001$, respectively).

For multivariable logistic regression analysis, the intermediate group was assigned as the reference category. We chose the variables that commonly affect cardiovascular clinical outcomes such as age, sex, etc. as regression models. In age and sex-adjusted analyses for all events, compared with the intermediate group, individuals in the late relative to those in early groups were at $46 \%$ higher risk of all events ( $\mathrm{OR}=1.46,95 \% \mathrm{CI}: 1.21$ to $1.77, \mathrm{P}=0.000)$. Further adjusted for LOS time slightly diminish the effect sizes $(\mathrm{OR}=1.23,95 \% \mathrm{CI}: 1.01$ to $1.50, \mathrm{P}=0.041)$. After further adjustment for potential confounders (BMI, Killip class II-III, smoking, history of cerebrovascular disease, diabetes mellitus, dyslipidemia, hypertension or infarct related artery), the association remained significant ( $\mathrm{OR}=1.26,95 \%$ CI: 1.02 to $1.56, \mathrm{P}=0.029$ ). No significantly increased risks for all events were observed for very early or early group individuals in all analyses, compared with intermediate group individuals. Similar results were observed for rates of secondary events and heart failure, but not for those of primary events and sudden cardiac death (Table 2).

Subgroups analyses were performed based on important baseline information from the whole study (Figures 1-7). For patients under the age of 65 or 65 and older, no significant differences in rates of in-hospital events were observed among the 4 groups (Figure 2). The intermediate group (PCI on days 7-14) also was defined as the reference category for subgroup analyses. The cumulative rate for all events during hospitalization in late $v s$. intermediate group was higher for women $(\mathrm{OR}=1.67,95 \% \mathrm{CI}$ : 1.07 to $2.62, \mathrm{P}=0.024)$ (Figure 1), but not for patients with heart failure (Killip class II or III; OR $=1.02,95 \%$ CI: 0.74 to $1.40, \mathrm{P}=0.908$ ) (Figure 6). Higher rate of all events during hospitalization 
Table 1 Baseline characteristics and in-hospital outcomes by delayed PCI time group

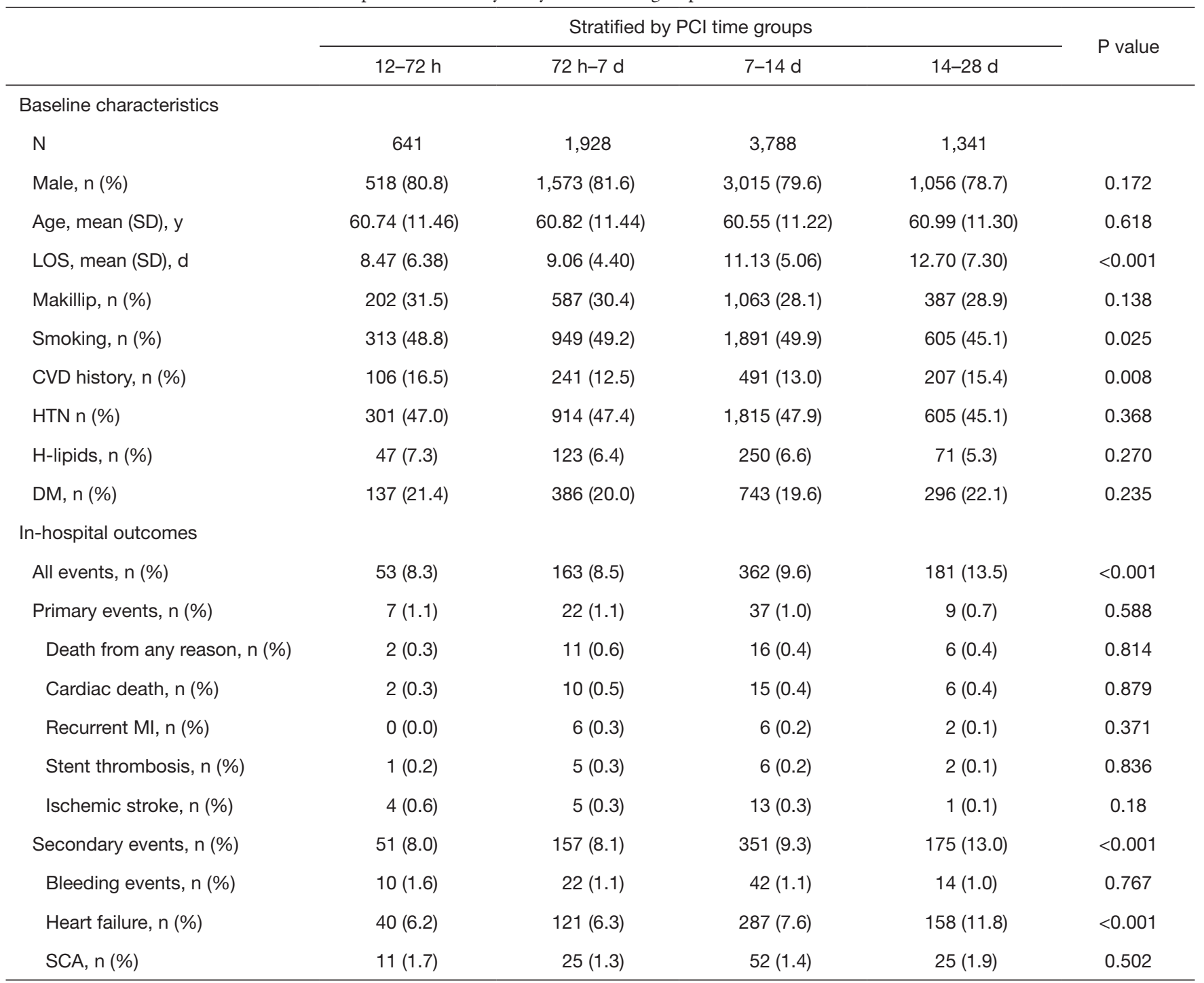

Data are expressed as mean (SD) or number (percentage). LOS indicates length of stay; MaKillip, killip class II or III; CVD, cerebral vascular disease; HBP, high blood pressure; H-lipids, hyperlipidemia; DM, diabetes mellitus; MI, myocardial infarction; SCA, sudden cardiac arrest.

were observed in late group for patients without diabetes mellitus, compared with intermediate group $(\mathrm{OR}=1.29$, $95 \%$ CI: 1.00 to $1.66, \mathrm{P}=0.048$ ) (Figure 3). Similar results were observed for patients without hypertension ( $\mathrm{OR}=1.42,95 \%$ CI: 1.05 to $1.92, \mathrm{P}=0.023$ ) (Figure 4), but not for patients with diabetes mellitus or hypertension (Figures 3,4). For patients with/without cerebrovascular disease, no significant differences in rates of in-hospital all events were observed among the 4 groups (Figure 5). Moreover, smokers undergoing late $v s$. intermediate delayed PCI (on days 14-28) were at $46 \%$ higher risk for all events
$(\mathrm{OR}=1.46,95 \% \mathrm{CI}: 1.05$ to $2.02, \mathrm{P}=0.023)$ (Figure 7).

\section{Discussion}

Previous studies have proven that final infarct size in STEMI patients with late reperfusion $(>12 \mathrm{~h})$ is larger than in patients with primary angioplasty $(<12 \mathrm{~h})$. However, substantial myocardial salvage can be obtained beyond the 12 hours limit, even when the infarct-related artery is totally occluded (11). For these reasons, early reperfusion therapy for patients with STEMI is indicated if the duration 
Table 2 Association between PCI time and clinical events

\begin{tabular}{|c|c|c|c|c|c|c|c|c|}
\hline Outcomes & $\begin{array}{l}\text { PCl time } \\
\text { groups }\end{array}$ & $\begin{array}{l}\text { Number of } \\
\text { events (\%) }\end{array}$ & \multicolumn{2}{|l|}{ Model 1} & \multicolumn{2}{|l|}{ Model 2} & \multicolumn{2}{|c|}{ Model 3} \\
\hline \multirow[t]{3}{*}{ All events } & $12-72 \mathrm{~h}$ & $53(8.3)$ & $0.84(0.62,1.14)$ & 0.272 & $0.97(0.71,1.33)$ & 0.863 & $0.89(0.64,1.23)$ & 0.470 \\
\hline & $72 \mathrm{~h}-7 \mathrm{~d}$ & $163(8.5)$ & $0.86(0.71,1.05)$ & 0.139 & $1.00(0.82,1.22)$ & 0.991 & $0.98(0.79,1.20)$ & 0.805 \\
\hline & $7-14 d$ & $362(9.6)$ & Reference & & Reference & & Reference & \\
\hline \multirow[t]{4}{*}{ Primary events } & $12-72 \mathrm{~h}$ & $7(1.1)$ & $1.11(0.49,2.50)$ & 0.803 & $1.18(0.52,2.67)$ & 0.689 & $1.09(0.48,2.48)$ & 0.845 \\
\hline & $72 \mathrm{~h}-7 \mathrm{~d}$ & $22(1.1)$ & $1.17(0.69,1.98)$ & 0.573 & $1.27(0.74,2.17)$ & 0.387 & $1.27(0.74,2.19)$ & 0.391 \\
\hline & $7 d-14 d$ & $37(1.0)$ & Reference & & Reference & & Reference & \\
\hline & $14-28 d$ & $9(0.7)$ & $0.67(0.32,1.40)$ & 0.290 & $0.63(0.30,1.31)$ & 0.213 & $0.65(0.31,1.36)$ & 0.250 \\
\hline \multirow{2}{*}{ Secondary events } & $7-14 d$ & $351(9.3)$ & Reference & & Reference & & Reference & \\
\hline & $14-28 d$ & $175(13.0)$ & $1.46(1.20,1.77)$ & 0.000 & $1.22(1.00,1.49)$ & 0.056 & $1.24(1.01,1.54)$ & 0.045 \\
\hline \multirow[t]{4}{*}{ Heart failure } & $12-72 \mathrm{~h}$ & $40(6.2)$ & $0.8(0.57,1.13)$ & 0.208 & $0.91(0.64,1.3)$ & 0.612 & $0.84(0.58,1.22)$ & 0.367 \\
\hline & $72 \mathrm{~h}-7 \mathrm{~d}$ & $121(6.3)$ & $0.81(0.65,1.00)$ & 0.055 & $0.93(0.75,1.17)$ & 0.554 & $0.91(0.71,1.15)$ & 0.427 \\
\hline & $7-14 d$ & $287(7.6)$ & Reference & & Reference & & Reference & \\
\hline & $14-28 d$ & $158(11.8)$ & $1.61(1.31,1.98)$ & $<0.001$ & $1.35(1.09,1.68)$ & 0.006 & $1.38(1.09,1.74)$ & 0.007 \\
\hline
\end{tabular}

Model 1 adjusted age, sex; Model 2 further adjusted LOS time; Model 3 further adjusted BMI, MaKillip, smoking, history of CVD or DM or dyslipidemia or hypertension, SBP, DBP, glucose, TC, HDL, LDL, TG and IRA. LOS, length of stay; BMI (Body mass index); MaKillip, killip II or III; CVD, cerebral vascular disease; DM, diabetes mellitus; SBP, systolic pressure; DBP, diastolic pressure; TC, total cholesterol; HDL, high density lipoprotein; LDL, low density lipoprotein; TG, triglyceride; IRA, infarct related artery.

\begin{tabular}{|c|c|c|c|c|c|}
\hline Subgroups & $\begin{array}{l}\mathrm{PCl} \text { time } \\
\text { groups }\end{array}$ & $\begin{array}{l}\text { Number of } \\
\text { events (\%) }\end{array}$ & OR $(95 \% \mathrm{Cl})$ & & $P$ value \\
\hline \multicolumn{6}{|l|}{ Age $<65 y$} \\
\hline \multirow[t]{4}{*}{ All events } & $12 \mathrm{~h}-72 \mathrm{~h}$ & $20(4.9)$ & $0.73(0.44-1.22)$ & -1 & 0.231 \\
\hline & $72 \mathrm{~h}-7 \mathrm{~d}$ & $71(5.8)$ & $0.84(0.61-1.14)$ & 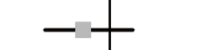 & 0.261 \\
\hline & $7 d-14 d$ & $171(7.1)$ & Reference & & Ref \\
\hline & $14-28 d$ & $86(10.5)$ & $1.29(0.95-1.74)$ & & 0.108 \\
\hline \multicolumn{6}{|l|}{ Age $\geq 65 y$} \\
\hline \multirow[t]{5}{*}{ All events } & $12 \mathrm{~h}-72 \mathrm{~h}$ & $33(14.1)$ & $0.98(0.63-1.54)$ & & 0.933 \\
\hline & $72 \mathrm{~h}-7 \mathrm{~d}$ & $92(12.9)$ & $1.1(0.82-1.47)$ & & 0.544 \\
\hline & $7 d-14 d$ & $191(14.0)$ & Reference & 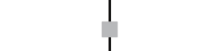 & Ref \\
\hline & $14-28 d$ & $95(18.2)$ & $1.18(0.86-1.61)$ & & 0.297 \\
\hline & & & & 1 & \\
\hline
\end{tabular}

Figure 2 Subgroup analyses for all in-hospital events by age. 


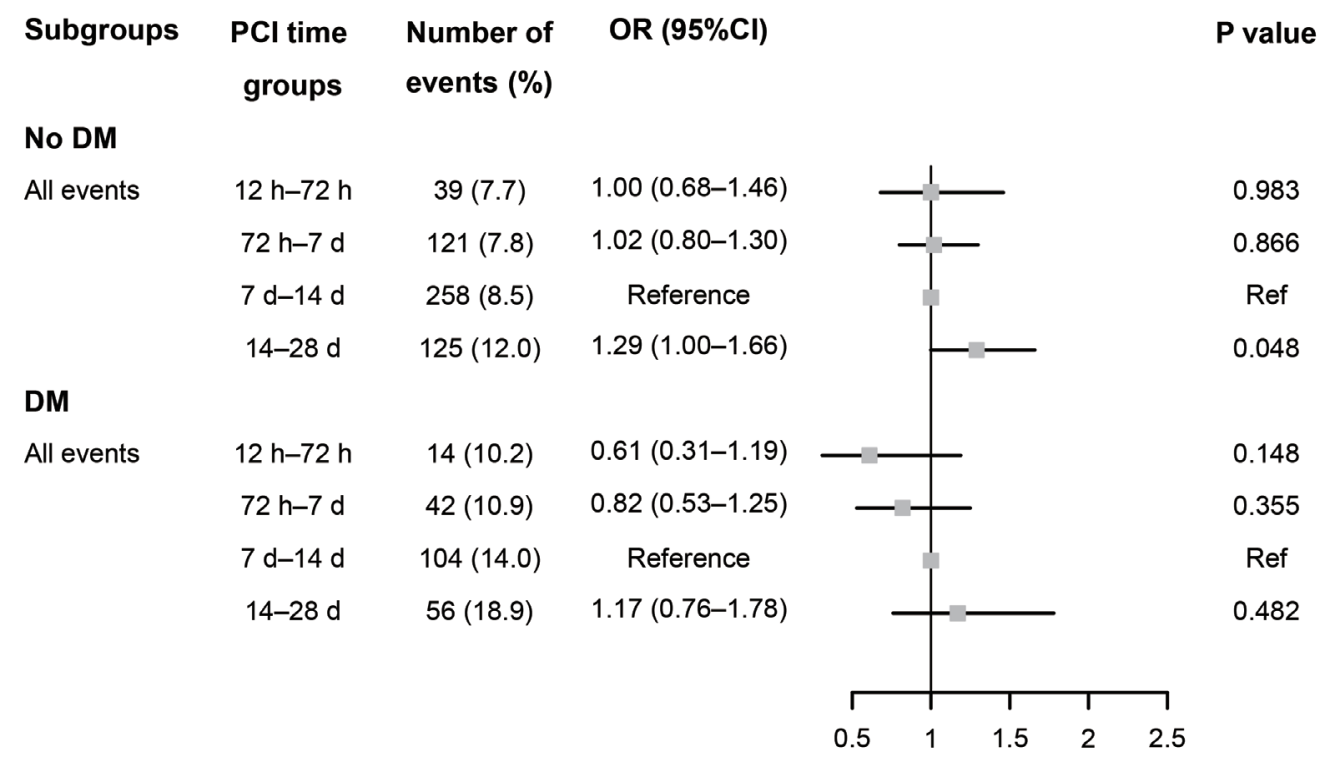

Figure 3 Subgroup analyses for all in-hospital events by presence/absence of diabetes mellitus.

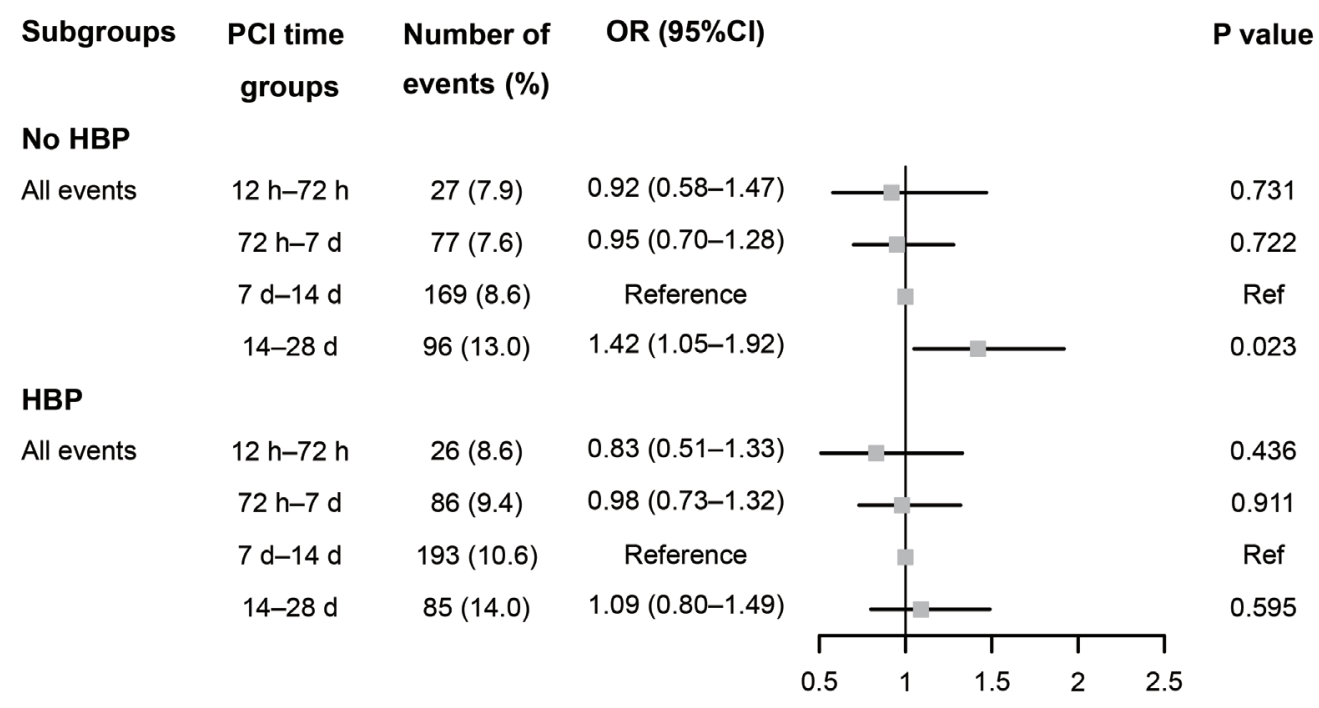

Figure 4 Subgroup analyses for all in-hospital events by presence/absence of hypertension.

of symptoms is $<12$ hours by the guidelines from the European and American societies of Cardiology. In patients with time from symptoms onset $>12$ hours, primary PCI is still recommended in the presence of continuing ischemia, life threatening-arrhythmia or if pain and ECG changes have been stuttering $(3,12)$.

The thrombotic environment within 12-48 hours of acute myocardial infarction would be anticipated to result in reduced coronary blood flow during coronary intervention, with an incidence of $50 \%(5,13)$. The main mechanism is MVO, and primary segments with MVO showed late wall thinning and no functional recovery at five months (14). The Beyond 12 hours Reperfusion Alternative Evaluation (BRAVE-2) trial showed a significant reduced area of myocardial infraction in patients treated with PCI (within 12-48 hours) compared with conservative therapy (6). Korea Acute Myocardial Infraction Registry (KAMIR) Investigators evaluated the benefit of PCI in stable early 


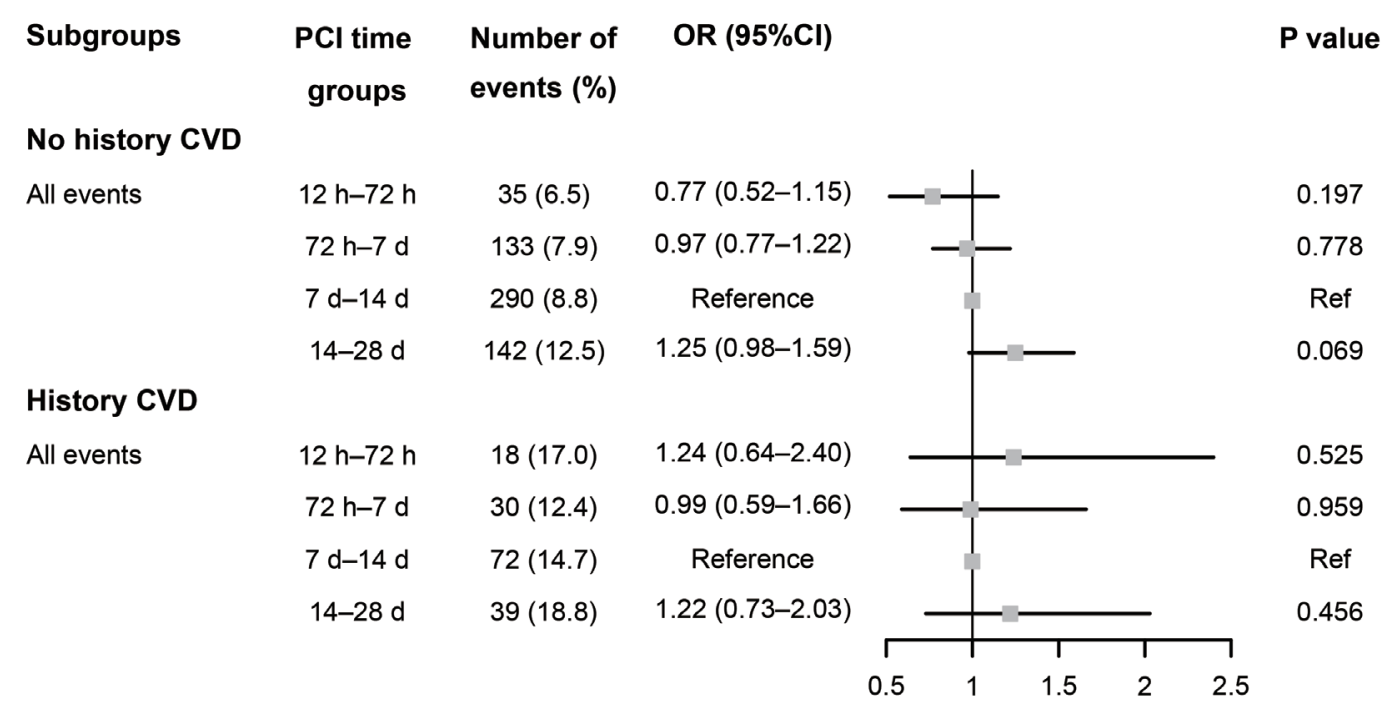

Figure 5 Subgroup analyses for all in-hospital events by presence/absence of cerebrovascular disease.

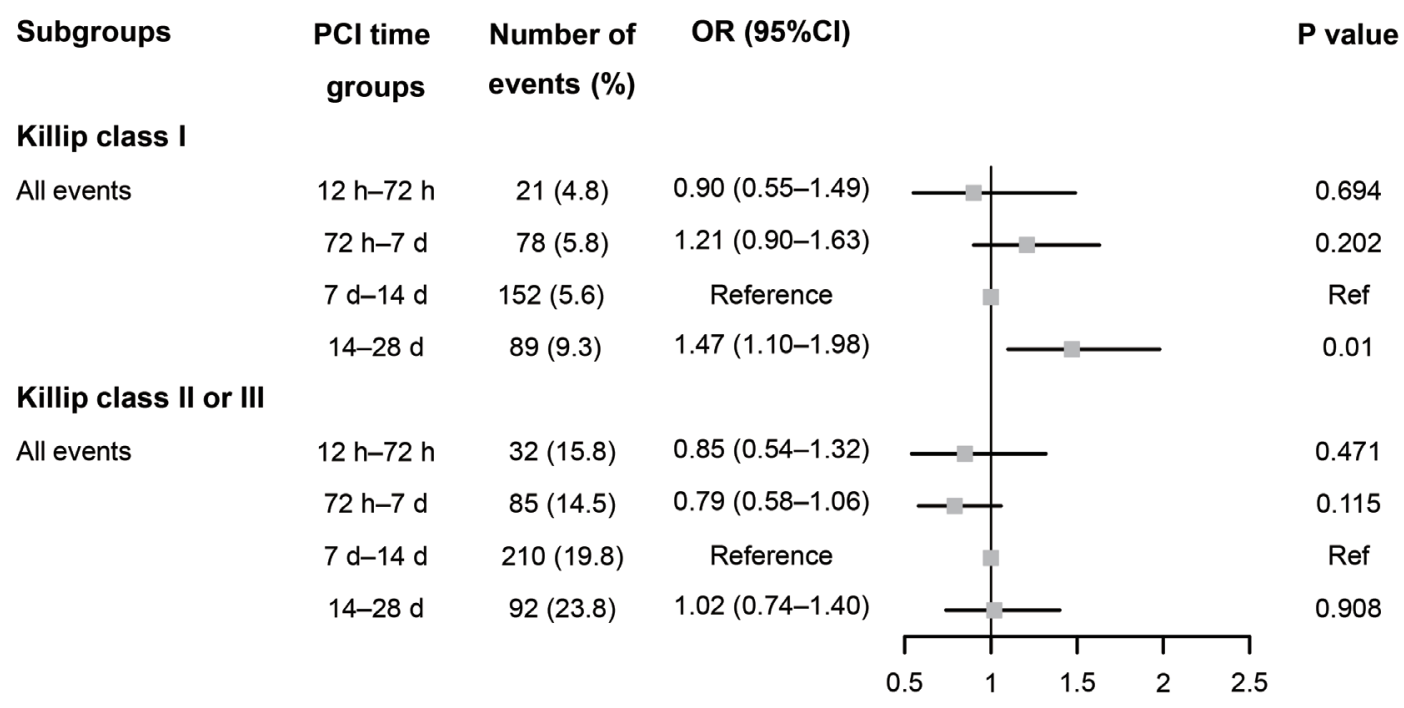

Figure 6 Subgroup analyses for all in-hospital events by Killip class.

latecomers with STEMI presenting 12-72 hours after symptom onset; PCI was associated with lower in-hospital mortality and improvement in the 12 -month clinical outcomes (7). In the Prospective National Observational Study (PL-ACS) on 2,306 hemodynamically stable patients with STEMI presenting 12 to 24 hours after symptom onset, patients undergoing an invasive approach had lower mortality after 12 months than patients with conservative treatment, which supports the idea of late reperfusion of STEMI (8). In addition, Abbate et al.'s meta-analysis demonstrated that PCI of the infarct-related artery (IRA) performed late (12 h to 60 days) after AMI is associated with significant improvements in cardiac function and survival (9). However, Yang et al.'s meta-analysis showed that PCI performed $>12 \mathrm{~h}$ but not 2-60 days after AMI is associated with significant improvement in clinical outcomes (15). Moreover, OAT demonstrated that routine PCI for a totally occluded IRA 3-28 days after acute MI failed to reduce 5 -year mortality, reinfarction, and severe heart failure (10). It should be noted that patients with 


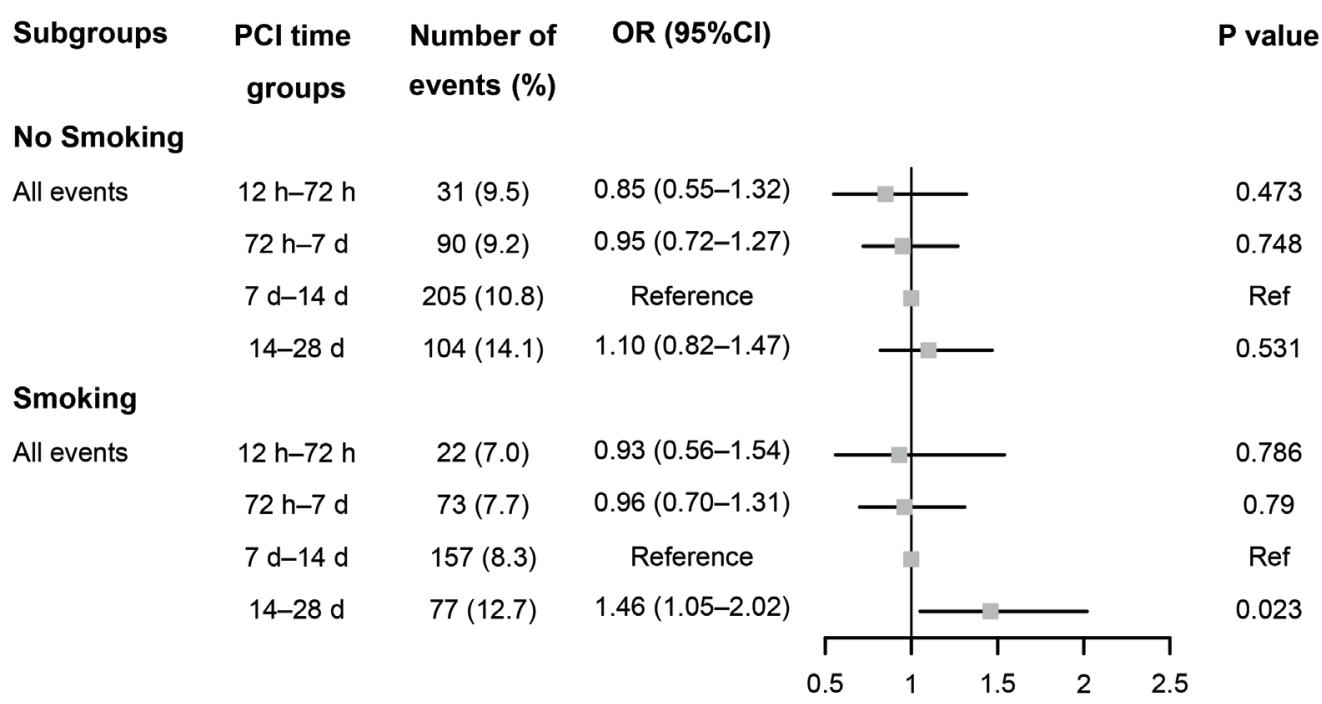

Figure 7 Subgroup analyses for all in-hospital events by smoking status.

hemodynamic instability were excluded from the latter study. Therefore, for the patients who missed the primary reperfusion time of STEMI, with hemodynamic stability, when to perform delayed revascularization remains controversial.

The purpose of our study was to assess the efficacy during hospitalization of delayed stenting in patients with STEMI within 12 hours to 28 days after symptoms onset. Patients with hemodynamic instability were excluded. All the patients were divided into four groups according to timing of revascularization. The study showed that patients with late PCI (14-28 days) had higher in-hospital rate of secondary outcomes especially heart failure but not of the primary outcome of MACE. Zheng et al. (16) also investigated STEMI patients who missed out on early reperfusion and underwent delayed PCI (2-28 days after PCI). They found delayed PCI at 15-28 days was associated with higher rate of 1-year MACE. The subgroup analysis of our research revealed that women had worse in-hospital outcomes in late group (14-28 days). This was consistent with the subgroup analysis of the OAT in which women had a higher primary endpoint event rate than did men, mainly driven by heart failure (17), which might be explained by women with STEMI being generally older, with more clustering risk factors than men, and less likely to present with ST-segment elevation, which would be anticipated to result in patient delay and worse outcomes (17-19). However, female patients included in CCC project are underrepresented. Future researches from larger studies is needed for a deep understanding of women with STEMI and delayed PCI. The other subgroup analyses showed that smokers in the late delayed PCI group (14-28 days) experienced a significant higher risk of all events during hospitalization. However, advanced age and comorbidities such as diabetes mellitus, hypertension and cerebrovascular disease were not associated with a risk disadvantage for the late group. We do not have an explanation for this finding. Interestingly, in STEMI patients with Killip class II or III on admission, early delayed PCI ( 12 h to $14 \mathrm{~d}$ ) was not associated with a significant benefit of in-hospital outcomes, suggesting that it might be safe to use medical therapy to improve cardiac function before PCI during hospitalization. However, long-term prognosis is unpredictable given the limitations of this study.

\section{Limitations}

The present study has the limitations inherent to the multicenter, observational cohort study design of the CCC project, and it cannot replace a randomized controlled trial. Data were collected from different regions and hospitals throughout China, rendering inevitable inherent uncontrolled differences in clinical practice among hospitals and geographical regions. Details on PCI procedures such as the TIMI blood flow during PCI were unavailable. In addition, the present study only assessed rates of adverse events during hospitalization, Therefore, the results need to be verified in clinical larger prospective studies with long- 
term follow-up.

\section{Conclusions}

In conclusion, late delayed PCI (14-28 days) after STEMI was associated with a higher incidence of adverse events particularly among women and smokers but not individuals with advanced age or comorbidities such as diabetes mellitus, hypertension and cerebrovascular disease. Moreover, it would appear safe for STEMI patients with heart failure (Killip class II-III) to be treated medically to improve cardiac function before delayed PCI.

\section{Acknowledgments}

We are grateful to all investigators in participating hospitals for their contributions to the project. The CCC-ACS project is a collaborative study by the American Heart Association (AHA) and Chinese Society of Cardiology. The AHA has been funded by Pfizer for quality improvement initiatives through an independent grant for learning and change.

\section{Footnote}

Conflicts of Interest: The authors have no conflicts of interest to declare.

Ethical Statement: The authors are accountable for all aspects of the work in ensuring that questions related to the accuracy or integrity of any part of the work are appropriately investigated and resolved. The study was approved by the Institutional Review Boards of participating hospitals and all patients provided written informed consent.

\section{References}

1. Keeley EC, Boura JA, Grines CL. Primary angioplasty versus intravenous thrombolytic therapy for acute myocardial infarction: a quantitative review of 23 randomised trials. Lancet 2003;361:13-20.

2. Christian TF, Schwartz RS, Gibbons RJ. Determinants of infarct size in reperfusion therapy for acute myocardial infarction. Circulation 1992;86:81-90.

3. Authors/Task Force members, Windecker S, Kolh P, et al. 2014 ESC/EACTS Guidelines on myocardial revascularization: The Task Force on Myocardial
Revascularization of the European Society of Cardiology (ESC) and the European Association for CardioThoracic Surgery (EACTS)Developed with the special contribution of the European Association of Percutaneous Cardiovascular Interventions (EAPCI). Eur Heart J 2014;35:2541-619.

4. Jaffe R, Dick A, Strauss BH. Prevention and treatment of microvascular obstruction-related myocardial injury and coronary no-reflow following percutaneous coronary intervention: a systematic approach. JACC Cardiovasc Interv 2010;3:695-704.

5. McDermott K, Maynard C, Trivedi R, et al. Factors associated with presenting $>12$ hours after symptom onset of acute myocardial infarction among Veteran men. BMC Cardiovasc Disord 2012;12:82.

6. Parodi G, Ndrepepa G, Kastrati A, et al. Ability of mechanical reperfusion to salvage myocardium in patients with acute myocardial infarction presenting beyond 12 hours after onset of symptoms. Am Heart J 2006;152:1133-9.

7. Sim DS, Jeong MH, Ahn Y, et al. Benefit of percutaneous coronary intervention in early latecomers with acute STsegment elevation myocardial infarction. Am J Cardiol 2012;110:1275-81.

8. Gierlotka M, Gasior M, Wilczek K, et al. Reperfusion by primary percutaneous coronary intervention in patients with ST-segment elevation myocardial infarction within 12 to 24 hours of the onset of symptoms (from a prospective national observational study [PL-ACS]). Am J Cardiol 2011;107:501-8.

9. Abbate A, Biondi-Zoccai GG, Appleton DL, et al. Survival and cardiac remodeling benefits in patients undergoing late percutaneous coronary intervention of the infarct-related artery: evidence from a meta-analysis of randomized controlled trials. J Am Coll Cardiol 2008;51:956-64.

10. Menon V, Pearte CA, Buller CE, et al. Lack of benefit from percutaneous intervention of persistently occluded infarct arteries after the acute phase of myocardial infarction is time independent: insights from Occluded Artery Trial. Eur Heart J 2009;30:183-91.

11. Busk M, Kaltoft A, Nielsen SS, et al. Infarct size and myocardial salvage after primary angioplasty in patients presenting with symptoms for $<12 \mathrm{~h}$ vs. $12-72 \mathrm{~h}$. Eur Heart J 2009;30:1322-30.

12. Endorsed by the Latin American Society of Interventional C, Pci Writing C, Levine GN, et al. 2015 ACC/AHA/ SCAI focused update on primary percutaneous coronary intervention for patients with ST-elevation myocardial 
Infarction: An update of the 2011 ACCF/AHA/SCAI guideline for percutaneous coronary intervention and the 2013 ACCF/AHA guideline for the management of STelevation myocardial infarction: A report of the American College of Cardiology/American Heart Association Task Force on Clinical Practice Guidelines and the Society for Cardiovascular Angiography and Interventions. Catheter Cardiovasc Interv 2016;87:1001-19.

13. Schomig A, Mehilli J, Antoniucci D, et al. Mechanical reperfusion in patients with acute myocardial infarction presenting more than 12 hours from symptom onset: a randomized controlled trial. JAMA 2005;293:2865-72.

14. Baks T, van Geuns RJ, Biagini E, et al. Effects of primary angioplasty for acute myocardial infarction on early and late infarct size and left ventricular wall characteristics. J Am Coll Cardiol 2006;47:40-4.

15. Yang HT, Xiu WJ, Zheng YY, et al. Invasive reperfusion after 12 hours of the symptom onset remains beneficial in patients with ST-segment elevation myocardial infarction: Evidence from a meta-analysis of published data. Cardiol J
2019;26:333-42.

16. Zheng $\mathrm{W}$, Yu CM, Liu J, et al. Patients with ST-segment elevation of myocardial infarction miss out on early reperfusion: when to undergo delayed revascularization. J Geriatr Cardiol 2017;14:524-31.

17. Reynolds HR, Forman SA, Tamis-Holland JE, et al. Relationship of female sex to outcomes after myocardial infarction with persistent total occlusion of the infarct artery: analysis of the Occluded Artery Trial (OAT). Am Heart J 2012;163:462-9.

18. Milcent C, Dormont B, Durand-Zaleski I, et al. Gender differences in hospital mortality and use of percutaneous coronary intervention in acute myocardial infarction: microsimulation analysis of the 1999 nationwide French hospitals database. Circulation 2007;115:833-9.

19. Loboz-Grudzien K, Jaroch J. Women with acute coronary syndromes have a worse prognosis - why? The need to reduce 'treatment-seeking delay'. Cardiol J 2011;18:219-21.
Cite this article as: $\mathrm{Wu}$ JW, Hu H, Li D, Ma LK. In-hospital outcomes of delayed stenting in hemodynamically stable patients with ST-segment elevation myocardial infarction: the CCC (Care for Cardiovascular Disease in China) project. Cardiovasc Diagn Ther 2019;9(5):462-471. doi: 10.21037/ cdt.2019.08.10 